\title{
Performance Comparison of Inverted Modulation Formats
}

\author{
Mehmet Sönmez \\ Osmaniye Korkut Ata University \\ Engineering Faculty, Department of \\ Electrical and Electronics Engineering
}

\begin{abstract}
In this paper, we describe a performance comparison of inverted modulation techniques which are defined as Inverse Pulse Position Modulation (IPPM) and Inverted Manchester Code (IMC) schemes. For AWGN channel, we analyze the bit error rate performance of the techniques. According to simulation results, IMC scheme has better BER performance than that of I-PPM technique Moreover, IMC scheme is experimentally designed by using Field Programmable Gate Arrays (FPGA).
\end{abstract}

\section{General Terms}

Modulator, Transmitter, Demodulator, Receiver, Hardware, Software, Optical Communication.et. al.

\section{Keywords}

Inverse 4-Pulse Position Modulation (I-4PPM), Field Programmable Gate Arrays (FPGA), Inverted Manchester Code (IMC), wireless optical communication.

\section{INTRODUCTION}

In these days, a novel Manchester Code technique, which is referred as to IMC, is improved for optical communication systems [1]. The optical communication systems are very popular in nowadays, specifically Visible Light Communication systems. The optical systems carry data signal using the light instead of electromagnetic wave in radio communication. Hence, many modulation techniques are necessary for transmission of data signal using light energy.

One of the modulation techniques for optical communication systems is On-Off Keying scheme [2]. The On-Off Keying technique is on case when transmitted data signal is logical ' 1 '. Otherwise, it is off case. In this case, the data signal is logical ' $O$ ' level. One of the disadvantages of On-Off Keying is flickering problem. In order to solve this problem, a technique, which is Manchester Code technique, is integrated on On-Off Keying scheme [2]. The Manchester code method acts as a pulse position transmission method. According to logical level of the data signal, the modulated signal changes pulse's position.

The one of the most basic techniques is pulse position modulation technique. Recently, this modulation technique has been improved by using its dimmable characteristic. The variable pulse position modulation is one of the leading techniques from among the dimmable techniques [2, 3]. In order to increase bit number in one symbol, the M-ary variable pulse position modulation techniques has been improved [4].
Another pulse position modulation scheme is Inverse Pulse Position Modulation scheme. This scheme is proposed by inverting traditional pulse position technique. The filled slots in traditional PPM are empty slots in I-PPM scheme, or vice versa. In I-PPM scheme, empty slot is determined by using decimal value of binary data bits.

In the past, many researchers were focused on I-PPM technique. One of these papers is aim to provide longer transmission distance and better power tolerance compared with techniques based on Manchester Code [5]. In this paper, the inverse 4-PPM is selected for performance comparison. Compared with OOK and PPM, it is shown from another paper that I-PPM scheme is more successful with respect to stable supply of power [6]. In order to ensure $75 \%$ dimming, Inverse 4-PPM technique was selected to data transmission method for Vehicular Visible Light Communication systems [7].

IMC technique has $75 \%$ dimming level since empty slot time is equal to quarter of one bit period. Hence, we use inverse 4PPM technique to compare with IMC method. In inverse 4PPM, dimming level is equal to $75 \%$ dimming level. Moreover, IMC method is implemented to monitor real-time results on FPGA board.

The paper is organized as follows: the I-4PPM and IMC techniques are given in the section II. The section III introduces the experimental and simulation results IMC architecture and I-4PPM technique. The section V concludes the paper.

\section{INVERSE PULSE POSITION MODULATION AND IMC TECHNIQUES} In this section, we present the theoretical analysis of inverted 4-PPM technique. As previous mentioned, I-4PPM has $75 \%$ dimming level because of the empty slot. Hence, the position of empty slot is determined to generate inverse 4-PPM signal by using decimal values of binary data bits. the inverse 4 PPM signal can be defined as follow:

$I-4 P P M(t)=\left(\begin{array}{ll}0 & \text { for } t \in\left[\frac{i}{4} T_{b}, \frac{i+1}{4} T_{b}\right] \\ V & \text { elsewhere }\end{array}\right)$

where, $i$ and $T_{b}$ can be defined as decimal value of binary data bit and one symbol period, respectively. As shown in the figure, empty slot is determined according to case of the data bits. 

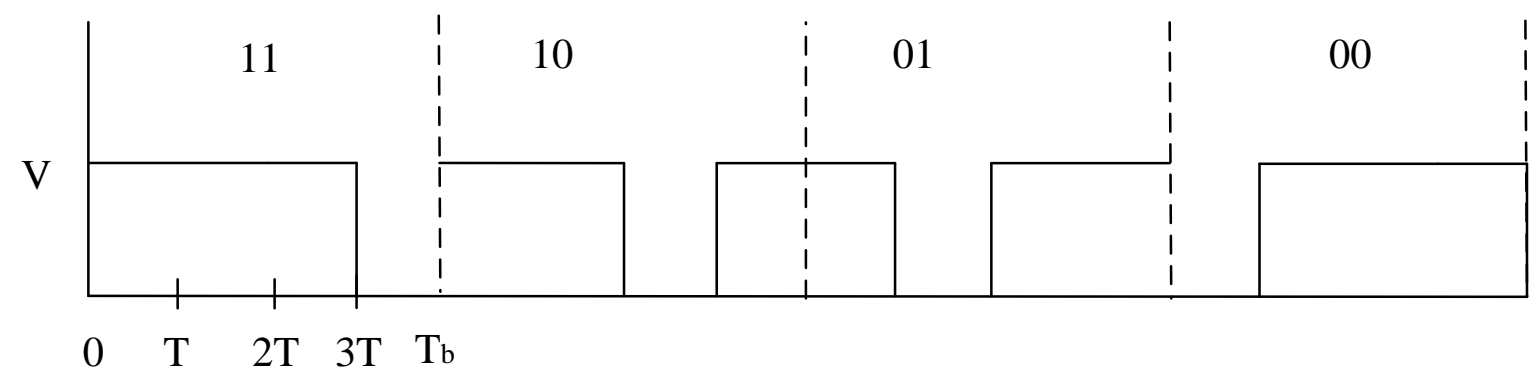

Fig 1: The waveform of I-4PPM

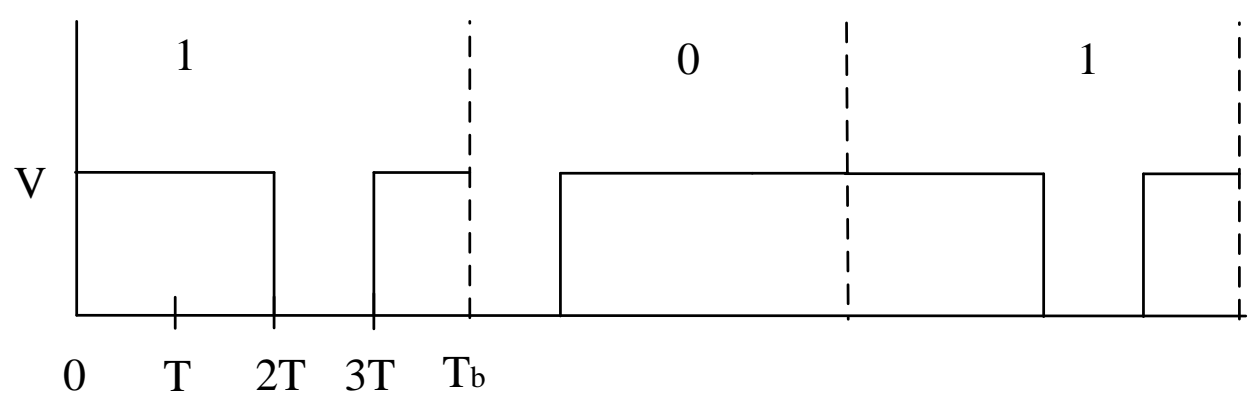

Fig 2: The waveform of IMC

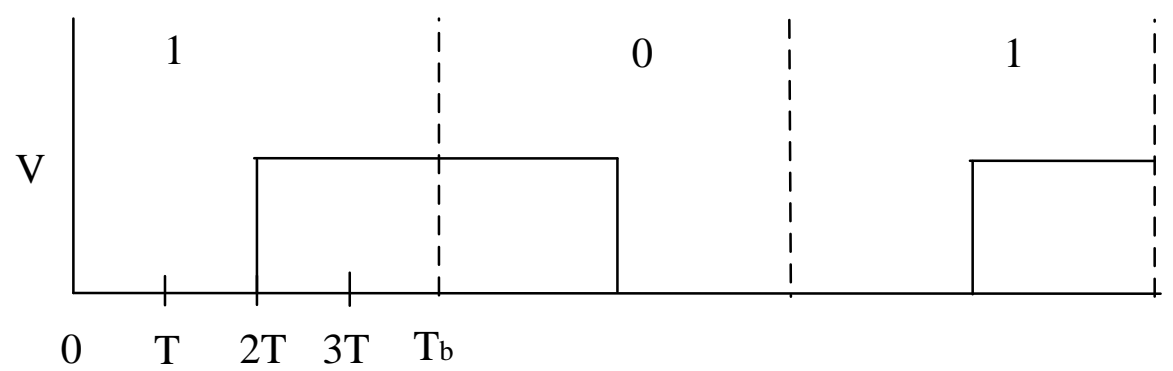

Fig 3: The waveform of Manchester Coded OOK

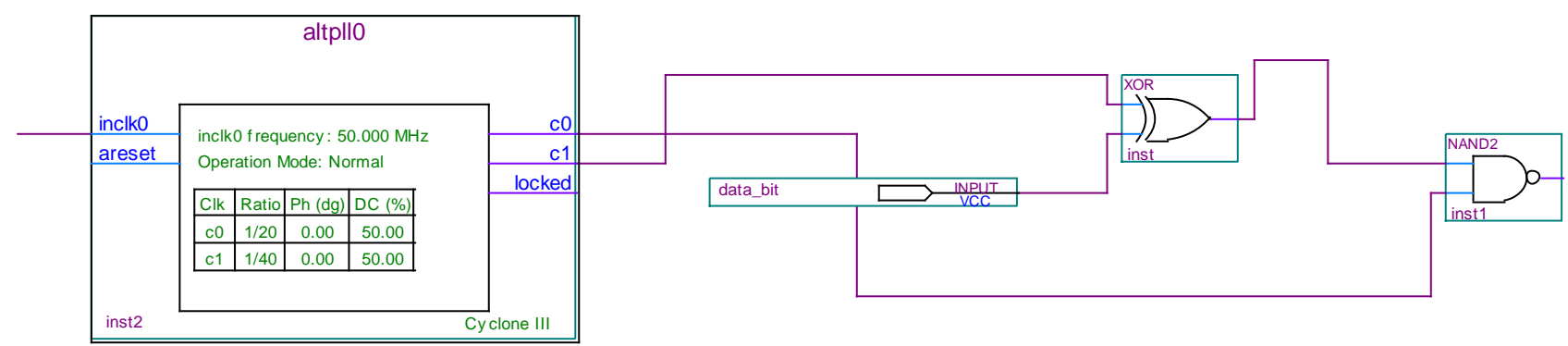

Fig. 4. The block scheme of IMC transmitter

In the Fig. 1, it is shown the changing of I-4PPM signal according to data bits. As shown in the figure, the decimal value of binary data signal determines the position of empty slot. In the Fig. 2, a waveform is given for IMC technique. The empty slot time is equal to that of I-4PPM while transmitted bit number of 4-IPPM method in one symbol period is further than that of IMC. In the Fig. 3, the traditional
Manchester Coded OOK signal is given. As shown in the figure, the logical ' 1 ' is transmitted by using the signal which is positioned at right side of symbol period while the logical ' 0 ' bit is sent by signal which is positioned at left side of the symbol period. Compared with IMC, the transmitted power is lesser than that of Manchester Coded Signal. However, performance analysis must be realized for bit error rate, 


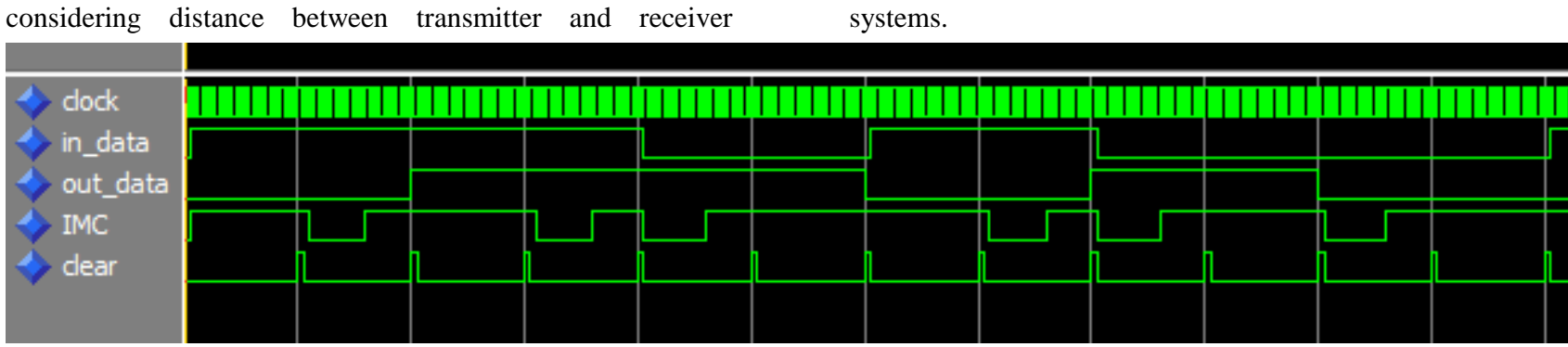

Fig 5: Simulation result of Inverted Manchester Code

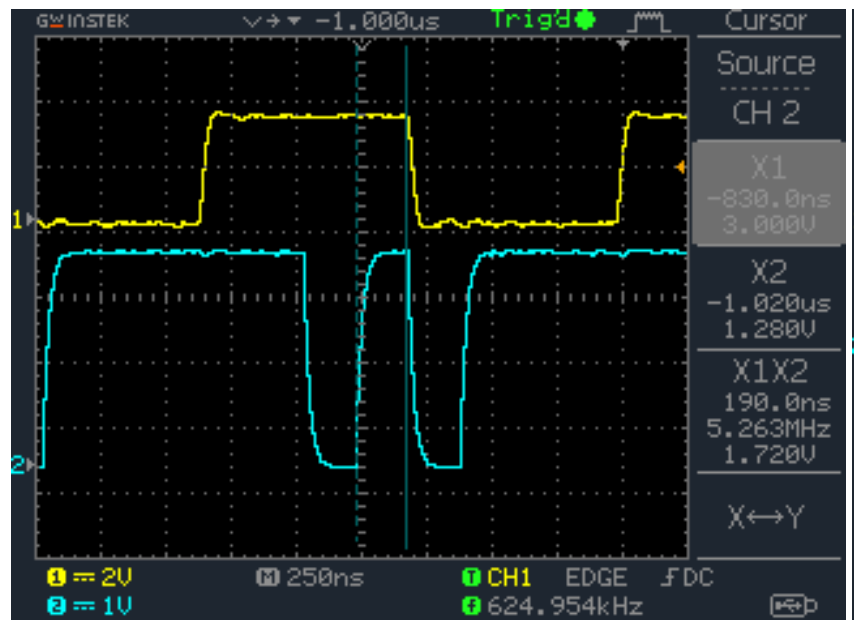

(a)

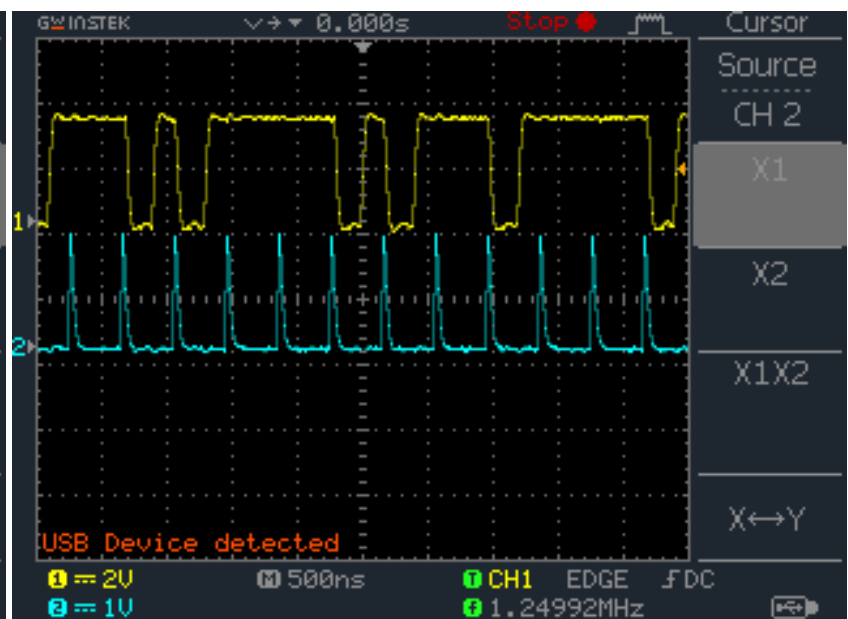

(b)

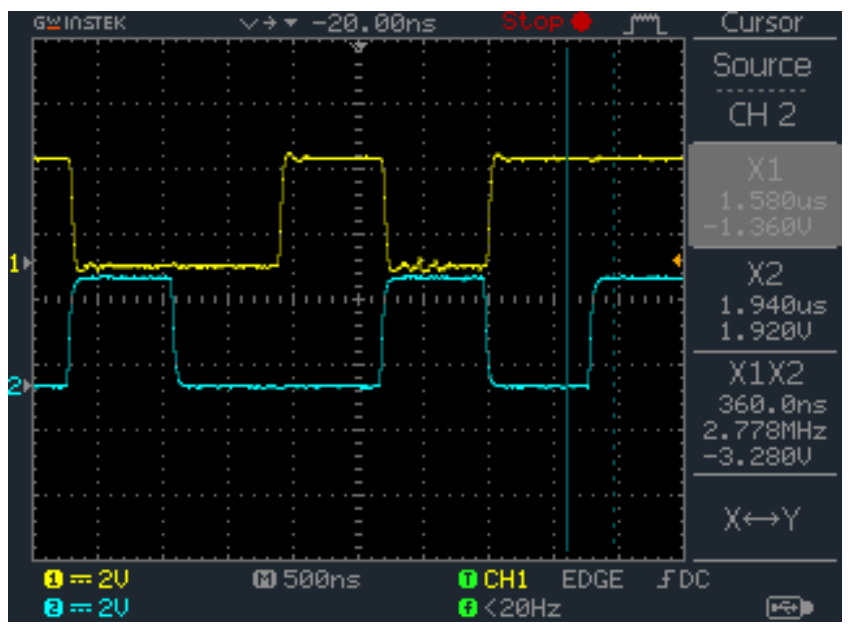

(c)

Fig 6: Experimental results of Inverted Manchester Code. (a) IMC signal according to data bits. (b) IMC signal with clear signal (c) Transmitted and demodulated data bits.

In the Fig. 4, a block scheme that is designed in FPGA complier program is given for IMC system. As presented in [1], the data signal and clock signal is applied on xor gate. it is important that the frequency of clock signal equal to twice of data rate. Thus, a phase locked loop (PLL) is used to generate appropriate signals. The output of xor gate is Manchester Coded signal that is shown in the Fig. 3. This signal is passed through a nand gate with a signal of which frequency equal to twice of clock signal. The output of nand gate generates IMC signal. Therefore, the IMC signal has $75 \%$ dimming level due to frequency of signal that is connected on nand gate

\section{SIMULATION AND EXPERIMENTAL RESULTS}

In this section, we give experimental and simulation results for IMC technique. According to simulation results in the Fig. 5 , inverted Manchester code signal is successfully generated. 


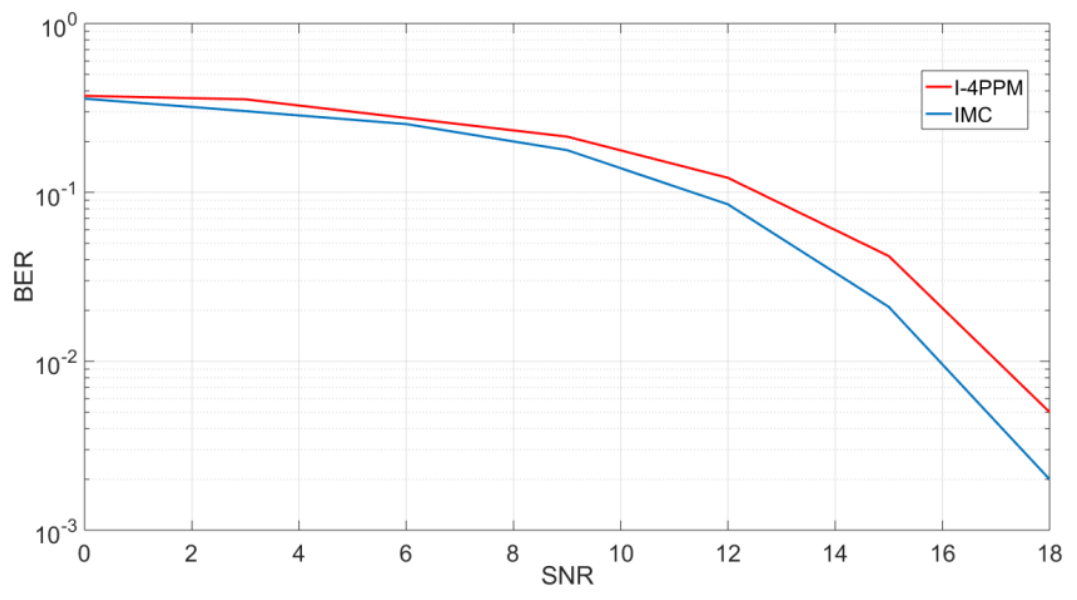

Fig 7: BER Comparison of I-PPM and IMC techniques.

IMC is inverted Manchester code signal. The clear is used to reset to the integrator. As shown in the Fig. 6, the logical ' 0 ' data bit, which is defined as yellow line, is encoded using "0111" bits. The logical ' 1 ' bit is encoded using "1101" bits. Compared with Fig. 2, the experimental result in Fig. 6a is successfully obtained. We also design a demodulator to estimate data bit from IMC signal. Hence, we use a integrator and a decision stage at the receiver side. In Fig. 6 b, the blue line shows the signal which clears the integrator block at each intervals. In the demodulator side, the decision block determines the slot of which integration value is the smallest. If the first slot is the smallest among four slots, the estimated bit is logical ' 0 ': However, the estimated bit will be logical ' 1 ' if the third slot has smallest value. The Fig. 6c illustrates the transmitted and demodulated data bits. The yellow line defines transmitted bits while blue line presents demodulated bits.

In the Fig. 7, BER performance of IMC and I-PPM signal is given. As shown in the figure, IMC has better performance than that of I-PPM.

\section{CONCLUSION}

In this paper, we give a performance comparisons of IMC and I-4PPM signals. According to simulation results, the BER performance of IMC is better than that of 4-IPPM. Additionally, we give a IMC structure by using FPGA complier. We demonstrate from experimental and simulation that designed IMC structure successfully generates data signal.

\section{REFERENCES}

[1] T. Shang, R. Chen, Y. Liu, ve Y. Gao, "Performance Analysis of IMC in FSO Systems Over Gamma-Gamma Channel", IEEE Photonics Journal, 10(3), pp. 1-13, Jun. 2018.

[2] IEEE Standard 802.15.7-2011, pp. 1-309, Jun. 2011.

[3] K. Lee ve H. Park, "Modulations for Visible Light Communications With Dimming Control", IEEE Photonics Technology Letters, 23(16), ss. 1136-1138, Aug. 2011.

[4] J. Yoo, B. W. Kim, ve S. Jung, "Modelling and analysis ofM-ary variable pulse position modulation for visible light communications", IET Optoelectronics, 9(5), pp. 184-190, 2015.

[5] Y. Lu ; S. Wang; L. Hu; X. Hong ; L. Xu, S. He. "Proposal of Inverse Pulse Position Modulation for Downstream Signal in Remodulation PON With PolSK-Modulated Multicast Overlay". IEEE Photonics Technology Letters, 24(12), pp. 1012-1014, 2012.

[6] R. K.; Y. Kozawa; Y. Umeda ; H. HabuchiInverse, "Pulse Position Modulation Schemes for Simultaneous Visible Light Wireless Information and Power Transfer", 2017 ITNAC, Nov. 2017.

[7] J.H. Yoo, R. Lee, J. K. Oh ; H.W. Seo, J.Y. Kim, H.C. Kim. S. Y. Jung. "Demonstration of Vehicular Visible Light Communication Based on LED Headlamp"2013 ICUFN, Jul. 2013 